\title{
Practical Use of Transient Elastography in Screening for Nonalcoholic Steatohepatitis in a Japanese Population
}

\author{
Haruka Hirono, Kazuhiko Watanabe, Katsuhiko Hasegawa and Shogo Ohkoshi* \\ Department of Internal Medicine, The Nippon Dental University School of Life Dentistry at Niigata, Chuo-ku, Niigata, Japan
}

\begin{abstract}
Background and Aims: Fatty infiltration of liver may induce insulin resistance (IR), and a proportion of patients with nonalcoholic fatty liver disease (NAFLD) is diagnosed with nonalcoholic steatohepatitis. Transient elastography is gaining popularity as a means of non-invasively determining both liver stiffness (fibrosis level) and degree of fatty infiltration, expressed as controlled attenuation parameter (CAP) value. Methods: The aims of this study were to investigate the association between IR and level of fatty liver, and to identify the group at a greater risk of nonalcoholic steatohepatitis using transient elastography and other noninvasive fibrosis markers. A total of 169 patients without chronic hepatitis B and C were analyzed. Results: The CAP value was significantly associated with IR (HOMA-IR $\geq 2.5$; AUROC $=0.81$ ), and the optimal cutoff to discriminate IR was $264 \mathrm{~dB} / \mathrm{m}$. The liver stiffness measurement and aspartate aminotransferase-to-platelet ratio index values were significantly higher for CAP $\geq 264$ than in CAP $<264$. The 9 patients among the overall 169 patients $(5.3 \%)$ and among the 102 NAFLD patients $(8.8 \%)$ who showed $\geq 264 \mathrm{~dB}$ and $\geq 7.0 \mathrm{kPa}$ in transient elastography could represent good candidates for liver biopsy. Conclusions: Evaluation of NAFLD based on CAP values was useful in diagnosing IR. About $9 \%$ of NAFLD patients in a Japanese outpatient clinic with a few metabolic complications might be considered good candidates for liver biopsy to confirm nonalcoholic steatohepatitis.

Citation of this article: Hirono $\mathrm{H}$, Watanabe $\mathrm{K}$, Hasegawa $\mathrm{K}$, Ohkoshi S. Practical use of transient elastography in screening for nonalcoholic steatohepatitis in a Japanese population. J Clin Transl Hepatol 2019;7(2):127-131. doi: 10.14218/JCTH.2018.00048.
\end{abstract}

\section{Introduction}

Nonalcoholic fatty liver disease (NAFLD) is the most common liver disease, estimated to affect up to about $30 \%$ of the

Keywords: NAFLD; Insulin resistance; NASH; Transient elastography; CAP; Fibrosis marker.

Abbreviations: APRI, aspartate aminotransferase-to-platelet ratio index; AST, aspartate aminotransferase; ALT, alanine aminotransferase; AUROC, area under the receiver operating characteristic curve; CAP, controlled attenuation parameter; HA, hyaluronic acid; IR, insulin resistance; LSM, liver stiffness measurement; M2BPGi, Mac-2 binding protein glycosylation isomer; NAFLD, nonalcoholic fatty liver disease; NASH, nonalcoholic steatohepatitis; ROC, receiver operating characteristic; T2DM, type 2 diabetes mellitus; TE, transient elastography.

Received: 22 August 2018; Revised: 16 May 2019; Accepted: 28 May 2019

*Correspondence to: Shogo Ohkoshi, Department of Internal Medicine, The Nippon Dental University School of Life Dentistry at Niigata, 1-8 Hamaura-cho, Chuo-ku, Niigata 951-8580, Japan. Tel: +81-25-211-8243, Fax: +81-25-2671582,E-mail: okoshi@ngt.ndu.ac.jp general population. ${ }^{1}$ NAFLD consists of simple steatosis, which accounts for the majority of cases, and $\mathrm{NASH}$, which results in liver cirrhosis and hepatocellular carcinoma. ${ }^{2,3}$ NAFLD is one of the signs of obesity and is highly correlated with metabolic syndrome, which includes type 2 diabetes mellitus (T2DM) and is characterized by insulin resistance (IR). IR may be induced by fat infiltrating the liver. ${ }^{4}$ So far, few studies have shown the relationship between levels of fatty infiltration of the liver and IR or the severity of T2DM.

Transient elastography (TE) (FibroScan ${ }^{\circledR}$, Echosens, Paris, France) is becoming popular as a routine and convenient technique for the diagnosis of liver diseases. ${ }^{5,6}$ This modality can assess hepatic stiffness as a liver stiffness measurement (LSM) and can quantify the degree of steatosis as a controlled attenuation parameter (CAP). Because the method is noninvasive and can be performed in a short time, it is well suited to population-based screening as a first-line method. ${ }^{7}$

The standard method to diagnose NASH among NAFLD is biopsy of the liver. Such histological findings as ballooning of hepatocytes, infiltration of lymphocytes and the presence of fibrosis allow the discrimination of NASH from simple steatosis. Because of the invasiveness of biopsy, alternate serological markers have been studied. ${ }^{8,9}$ In addition to LSM and CAP evaluations, many serological markers, including the aspartate aminotransferase (AST)-to-platelet ratio index (APRI), ${ }^{10} \mathrm{Fib}-4$ index, ${ }^{11}$ NAFLD fibrosis score, ${ }^{12}$ and hyaluronic acid (HA) have been evaluated for their utility in detecting liver fibrosis. ${ }^{9,13}$ Recently, the assay of Mac-2 binding protein glycosylation isomer (M2BPGi) has proven useful in the evaluation of liver fibrosis levels for various chronic liver diseases, including NASH. ${ }^{14-17}$ The values were clearly associated with liver fibrosis levels among NASH patients. ${ }^{14,16}$ However, few reports have attempted to verify its usefulness in an outpatient population with only a few metabolic complications.

This study examined the association between CAP values and IR. We have also attempted to estimate the incidence of NASH using TE and other fibrosis markers, including M2BPGi, among outpatients of our hospital.

\section{Methods}

\section{Patients}

This was a single-center study of patients carried out between October 2016 and March 2018. Demographic, clinical, and biochemical characteristics of the outpatient population are shown in Table 1. A total of 169 patients without excessive alcohol intake or chronic hepatitis B or C, who agreed to be enrolled in this study and to undergo TE and serum fibrosis tests were investigated. Thirty-two (19\%) of them were from 
among those who had an annual thorough medical checkup. Others $(81 \%)$ were those who were seen sequentially in the clinic during the above-mentioned period.

NAFLD was defined by the presence of fatty liver on ultrasonography and alcohol intake $<20 \mathrm{~g} /$ day. Based on the clinical diagnosis and medications in the medical charts, ongoing pharmacotherapy for hypertension, diabetes, hypercholesterolemia, hypertriglyceridemia, and hyperuricemia were seen in $42.0 \%, 17.2 \%, 25.4 \%, 8.9 \%$, and $5.9 \%$. In addition, $88(52.1 \%)$ patients had obstructive sleep apnea syndrome. CAP measurement software was installed with TE, and values were obtained during LSM assessment. ${ }^{6}$ Their values were expressed in $\mathrm{dB} / \mathrm{m}$.

This study was carried out in accordance with the ethical standards of the Declaration of Helsinki and was approved by the ethics committee of School of Life Dentistry at Niigata, The Nippon Dental University (ECNG-H-247).

\section{Statistical analysis}

Quantitative values were presented as mean \pm standard deviation. An unpaired $t$-test was used for comparing continuous

Table 1. Clinical backgrounds of the studied population, $n=169$

\begin{tabular}{ll}
\hline Characteristic & Results \\
\hline M / F & $100(59.2 \%) /$ \\
& $69(40.8 \%)$ \\
Hypertension -/+ & $98(58.0 \%) /$ \\
& $71(42.0 \%)$ \\
Type 2 diabetes mellitus -/+ & $140(82.8 \%) /$ \\
Hypercholesterolemia -/+ & $29(17.2 \%)$ \\
& $126(74.6 \%) /$ \\
Hypertriglyceridemia -/+ & $43(25.4 \%)$ \\
Hyperuricemia -/+ & $154(91.1 \%) /$ \\
Obstructive sleep apnea & $15(8.9 \%)$ \\
syndrome -/+ & $159(94.1 \%) /$ \\
Age (years) & $10(5.9 \%)$ \\
Body mass index, kg/m ${ }^{2}$ & $81(47.9 \%) /$ \\
Aspartate aminotransferase, IU/L & $88(52.1 \%)$ \\
Alanine aminotransferase, IU/L & $59.8 \pm 13.2$ \\
AST/ALT & $25.0 \pm 4.1$ \\
Gamma-glutamyl transpeptidase, & $26.3 \pm 11.0$ \\
IU/L & $32.7 \pm 22.8$ \\
Triglyceride, mg/dL & $0.95 \pm 0.33$ \\
Low-density lipoprotein cholesterol, & $40.8 \pm 37.6$ \\
mg/dL & $122.8 \pm 31.5$ \\
High-density lipoprotein cholesterol, & $59.9 \pm 16.2$ \\
mg/dL & \\
Uric acid, mg/dL & $5.36 \pm 1.21$ \\
Hemoglobin A1C, \% & $6.17 \pm 0.91$ \\
Highly sensitive-C-reactive protein, & $0.1179 \pm 0.1907$ \\
mg/dL & \\
\hline & \\
& \\
& \\
&
\end{tabular}

variables between groups for normally distributed data, and the Welch's $t$-test was applied for data displaying nonnormal distributions. Receiver operating characteristic (ROC) curves were constructed to assess the accuracy of CAP and to identify optimal cut-offs. Optimal cut-offs of CAP to elucidate the IR were chosen to maximize Youden's index. All analyses were carried out using the JMP software version 13.2.1 in statistical analysis SAS software.

\section{Results}

Table 1 shows the characteristics of the studied population ( $n=169)$. NAFLD was diagnosed in $102(60.4 \%)$ patients. Area under the ROC curve (AUROCs) for body mass index and degree of fatty infiltration (expressed as CAP values) for diagnosing insulin resistance (HOMA-IR $\geq 2.5$ ) were both 0.81 , showing a good correlation (Fig. 1). Cut-off values were $25.49 \mathrm{~kg} / \mathrm{m}^{2}$ for body mass index and $264 \mathrm{~dB} / \mathrm{m}$ for $\mathrm{CAP}$, respectively. Patients were divided into two groups using a cut-off of $264 \mathrm{~dB} / \mathrm{m}$, and clinical factors were compared (Table 2). Body mass index, AST, alanine aminotransferase, gamma-glutamyl transpeptidase, triglyceride, uric acid, hemoglobin A1c, and highly sensitive-C-reactive protein were significantly higher in the CAP $\geq 264 \mathrm{~dB} / \mathrm{m}$ group, and high-density lipoprotein cholesterol was lower in this group. Regarding liver fibrosis makers, APRI and LSM were significantly higher in the CAP $264 \mathrm{~dB} / \mathrm{m}$ group (0.432 vs. 0.336 and $5.05 \mathrm{kPa}$ vs. $3.92 \mathrm{kPa}$, respectively).

Based on the results of past reports that defined cut-off values for the presence of fibrosis at around $7.0 \mathrm{kPa}$ in NAFLD,${ }^{18,19}$ we tentatively extracted those patients showing values over these thresholds (CAP $\geq 264$, LSM $\geq 7.0$ ) to detect patients with suspected NASH. Nine patients $(5.3 \%$ of the total cohort of 169 patients, $8.8 \%$ of the NAFLD subgroup of 102 patients) fulfilled these criteria, and the results of other fibrosis tests for these nine patients are shown in Table 3. One to four serum markers of fibrosis showed abnormal values.

\section{Discussion}

Obesity and IR are the main causes of metabolic syndrome. NAFLD is closely associated with metabolic syndrome, and T2DM is associated with a higher rate of severe NAFLD. ${ }^{4}$ Liver fat is well known to induce IR, so a close association exists between the level of IR and severity of NAFLD. ${ }^{20}$ Values of HOMA-IR and CAP of TE reportedly increase, as people with NAFLD proceed from normal glucose tolerance to pre-diabetes mellitus and diabetes mellitus. ${ }^{21}$ We studied the relationship between levels of fatty liver and IR, using the CAP evaluation to which many studies have shown a clear correlation between CAP values and levels of steatosis obtained by biopsied samples. ${ }^{5,22,23}$ ROC curve analysis between CAP values and body mass index showed a significant correlation (AUROC $=0.81$; Fig. 1). We also observed a significant association between IR (HOMA-IR $\geq 2.5$ ) and CAP values (AUROC = 0.81 ) and found that the cut-off CAP value to best show IR was $264 \mathrm{~dB} / \mathrm{m}$.

Liu et $a l .{ }^{24}$ reported that the median CAP value for a large population was $250 \mathrm{~dB} / \mathrm{m}$. This value has been confirmed by several reports as a significant level for showing minimal hepatic steatosis in biopsy samples. ${ }^{5,25,26}$ For example, Karlas et al. ${ }^{5}$ reported that a CAP of $248 \mathrm{~dB} / \mathrm{m}$ corresponded to histologically confirmed steatosis. Interestingly, the 

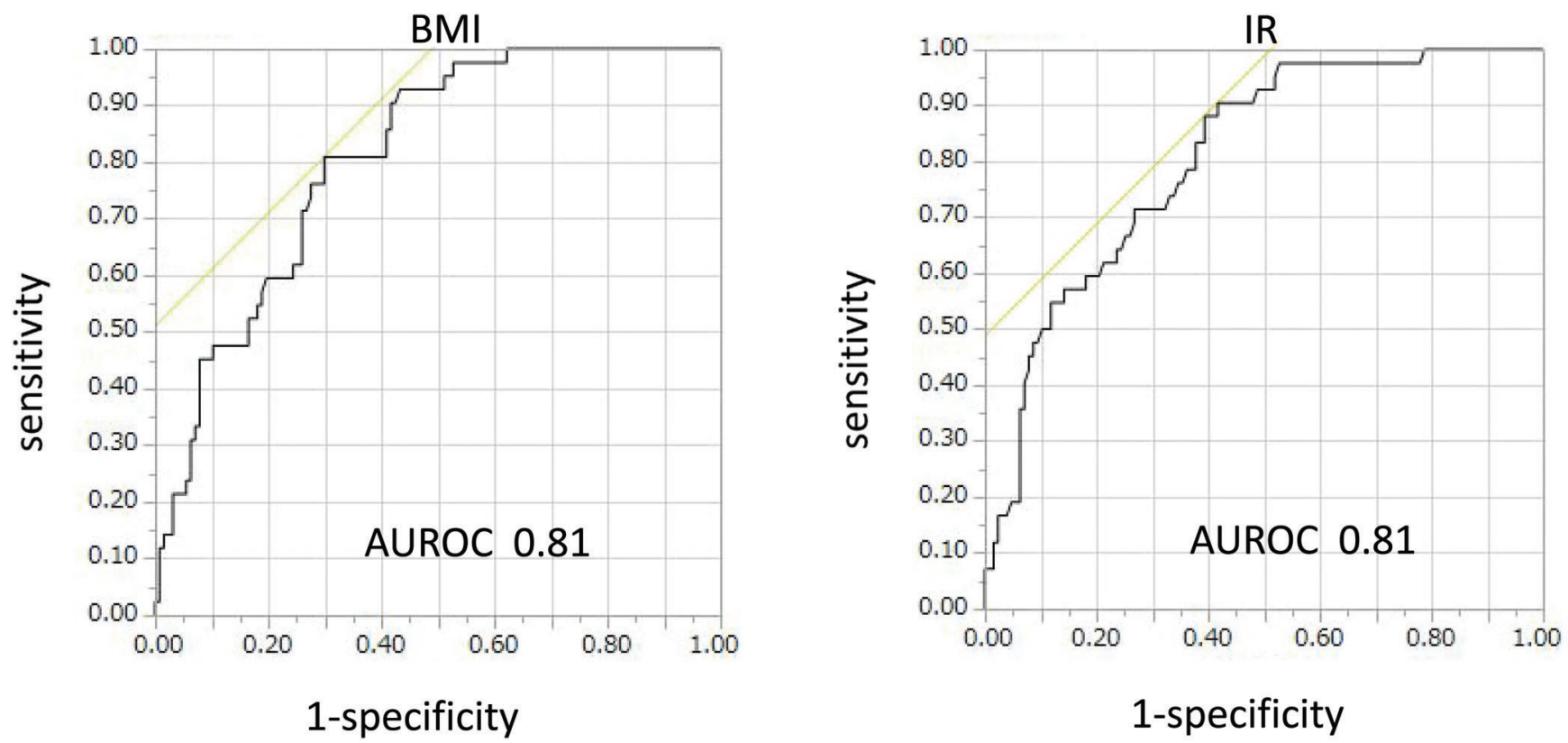

Fig. 1. Diagnostic capabilities of body mass index and controlled attenuation parameter values for assessing insulin resistance. AUROC values were 0.81 for both. AUROC of body mass index and controlled attenuation parameter values for diagnosing insulin resistance (HOMA-IR $\geq 2.5$ ). Abbreviation: AUROC, area under the receiver operating characteristic curve.

proximity of our cut-off index for CAP to reveal IR $(264 \mathrm{~dB} / \mathrm{m})$ to this value supports the close association between level of hepatic steatosis and presence of IR in our mostly (82.8\%) nondiabetic population.
Chon et al. ${ }^{21}$ also reported that median CAP values in subjects with T2DM was $265 \mathrm{~dB} / \mathrm{m}$, so the presence of small diabetic population in our study $(17.2 \%)$ may not have a great effect on our overall data. NAFLD is reportedly associated with

Table 2. Comparison of biochemical and fibrosis tests between individuals divided by controlled attenuation parameter value that showed insulin resistance (HOMA-IR $\geq 2.5$ )

\begin{tabular}{|c|c|c|c|}
\hline Controlled attenuation parameter, $\mathrm{dB} / \mathrm{m}$ & $<264, n=78$ & $\geqq 264, n=91$ & $p$-value \\
\hline Body mass index, $\mathrm{kg} / \mathrm{m}^{2}$ & $22.3 \pm 3.0$ & $27.3 \pm 3.6$ & $<0.001$ \\
\hline Aspartate aminotransferase, IU/L & $22.3 \pm 5.2$ & $29.7 \pm 13.4$ & $<0.001$ \\
\hline Alanine aminotransferase, IU/L & $21.7 \pm 8.3$ & $42.1 \pm 26.8$ & $<0.001$ \\
\hline AST/ALT & $1.10 \pm 0.29$ & $0.82 \pm 0.30$ & $<0.001$ \\
\hline Gamma-glutamyl transpeptidase, IU/L & $30.3 \pm 31.2$ & $49.8 \pm 40.4$ & $<0.001$ \\
\hline Triglyceride, mg/dL & $111.1 \pm 12.6$ & $160.0 \pm 11.6$ & $<0.01$ \\
\hline Low-density lipoprotein cholesterol, mg/dL & $120.0 \pm 29.6$ & $125.3 \pm 32.9$ & 0.267 \\
\hline High-density lipoprotein cholesterol, mg/dL & $66.4 \pm 18.1$ & $54.3 \pm 11.8$ & $<0.001$ \\
\hline Uric acid, mg/dL & $5.10 \pm 1.17$ & $5.58 \pm 1.21$ & $<0.05$ \\
\hline Hemoglobin A1c, \% & $5.90 \pm 0.64$ & $6.39 \pm 1.04$ & $<0.001$ \\
\hline Highly-sensitive-C-reactive protein, mg/dL & $0.0729 \pm 0.1560$ & $0.1526 \pm 0.2079$ & $<0.01$ \\
\hline Nonalcoholic fatty liver disease fibrosis score & $2.280 \pm 0.994$ & $2.161 \pm 1.369$ & 0.533 \\
\hline Aspartate aminotransferase-to-platelet ratio index & $0.336 \pm 0.125$ & $0.432 \pm 0.230$ & $<0.001$ \\
\hline Fibrosis-4 score & $1.45 \pm 0.08$ & $1.23 \pm 0.07$ & $<0.05$ \\
\hline Hyaluronic acid, ng/mL & $52.83 \pm 48.66$ & $46.93 \pm 63.66$ & 0.567 \\
\hline Type IV collagen $\cdot 7 \mathrm{~S}, \mathrm{ng} / \mathrm{mL}$ & $3.36 \pm 0.78$ & $3.38 \pm 0.86$ & 0.860 \\
\hline Mac-2 binding protein glycosylation isomer & $0.496 \pm 0.261$ & $0.559 \pm 0.419$ & 0.236 \\
\hline Liver stiffness measurement, $\mathrm{kPa}$ & $3.92 \pm 1.21$ & $5.05 \pm 2.71$ & $<0.001$ \\
\hline Interquartile range, $\%$ & $15.04 \pm 6.94$ & $14.39 \pm 6.40$ & 0.527 \\
\hline
\end{tabular}


Hirono $\mathrm{H}$. et al: TE, insulin resistance, and $\mathrm{NASH}$

Table 3. Fibrosis markers of 9 patients which showed more than $263 \mathrm{~dB} / \mathrm{m}$ and $7.0 \mathrm{kPa}$ by transient elastography

\begin{tabular}{|c|c|c|c|c|c|c|c|c|c|}
\hline Patient \# & 1 & 2 & 3 & 4 & 5 & 6 & 7 & 8 & 9 \\
\hline Age in years & 40 & 68 & 77 & 51 & 63 & 64 & 59 & 55 & 50 \\
\hline Sex & M & M & $\mathrm{F}$ & M & $\mathrm{F}$ & $\mathrm{F}$ & $\mathrm{F}$ & $\mathrm{F}$ & M \\
\hline Body mass index, $\mathrm{kg} / \mathrm{m}^{2}$ & 37.0 & 29.7 & 24.8 & 25.8 & 24.0 & 31.5 & 34.7 & 29.1 & 39.6 \\
\hline SAS +/- & + & - & + & - & - & + & - & + & - \\
\hline HOMA-IR & 11.4 & 8.5 & 11.3 & 8.6 & 3.1 & 4.6 & 5.1 & 2.0 & 7.3 \\
\hline $\begin{array}{l}\text { Nonalcoholic fatty liver disease } \\
\text { fibrosis score }\end{array}$ & $0.61^{*}$ & $0.06^{*}$ & 1.33 & 1.71 & 2.01 & 1.07 & ND & 1.99 & $0.52^{*}$ \\
\hline $\begin{array}{l}\text { Aspartate aminotransferase-to- } \\
\text { platelet ratio index }\end{array}$ & 0.63 & 1.29 & 1.33 & 0.82 & 0.15 & 0.51 & 0.57 & 1.16 & 0.40 \\
\hline Fibrosis-4 score & 0.93 & 2.22 & $4.79^{*}$ & 1.15 & 0.71 & 1.33 & 1.55 & 1.89 & 0.82 \\
\hline Hyaluronic acid, ng/mL & $110.6^{*}$ & $488.9^{*}$ & $116.8^{*}$ & $107.1^{*}$ & $109.3^{*}$ & $63.6^{*}$ & $179.0^{*}$ & 14.0 & 41.0 \\
\hline Type IV collagen $\cdot 7 \mathrm{~S}, \mathrm{ng} / \mathrm{mL}$ & 3.4 & $7.4^{*}$ & 5.3 & 4.2 & 2.8 & 3.2 & 4.3 & 5.0 & 4.6 \\
\hline $\begin{array}{l}\text { Mac- } 2 \text { binding protein glycosylation } \\
\text { isomer }\end{array}$ & 0.48 & $1.08^{*}$ & $1.25^{*}$ & 0.45 & 0.34 & 0.48 & $0.81^{*}$ & $3.99^{*}$ & 0.55 \\
\hline Liver stiffness measurement, $\mathrm{kPa}$ & 21.8 & 15.1 & 14.3 & 8.8 & 8.7 & 8.6 & 7.7 & 7.3 & 7.1 \\
\hline $\begin{array}{l}\text { Controlled attenuation parameter, } \\
\mathrm{dB} / \mathrm{m}\end{array}$ & 396 & 299 & 266 & 346 & 266 & 349 & 280 & 366 & 396 \\
\hline
\end{tabular}

Standard values: Nonalcoholic fatty liver disease fibrosis score $>0.676 ;{ }^{12}$ APRI $<1.85 ;{ }^{10}$ Fibrosis-4 $<2.67 ;{ }^{11}$ hyaluronic acid $<50 \mathrm{ng} / \mathrm{mL}$; Type IV collagen $\cdot 7 \mathrm{~S}<6 \mathrm{ng} / \mathrm{mL}$; M2BPGi $<0.70 .^{14}$

*Values that are not within these standards.

an increased frequency of $\mathrm{T}^{2} \mathrm{DM}^{27}$ and the abnormal CAP values may be involved in the initiation of IR. In our study, HOMA-IR gradually increased from $1.16 \pm 0.62$ in $<248 \mathrm{~dB} / \mathrm{mL}$ that was reported to be $<S 1,2.78 \pm 5.03$ in 248 to 267 to be $\mathrm{S} 1$, to $2.89 \pm 2.16$ in $>280 \mathrm{~dB}$ to be $\mathrm{S} 3 .{ }^{5}$

The reported incidence of NASH among NAFLD has been variable (2-20\%) because of differences in diagnosis methods and patients cohorts. ${ }^{2,28}$ In general, the incidence of NASH becomes higher as steatosis levels increase and is highly associated with the presence of diabetes mellitus. ${ }^{4,29,30}$ We compared background and clinical factors between groups separated by a CAP threshold of $264 \mathrm{~dB} / \mathrm{m}$, which allowed significant differentiation of IR in our study population. As a result, body mass index, aspartate aminotransferase, alanine aminotransferase, gamma-glutamyl transpeptidase, triglyceride, uric acid, hemoglobin A1c, and highly sensitive-C-reactive protein were significantly higher in the group with CAP $\geq 264$ $\mathrm{dB} / \mathrm{m}$, and high-density lipoprotein cholesterol was lower. Regarding fibrosis markers, LSM values and APRI were significantly higher in CAP $\geq 264 \mathrm{~dB} / \mathrm{m}$. However, no significant differences were seen in other serological markers, including $H A$, type IV collagen 7S, and M2BPGi.

LSM has been reported as useful for evaluating fibrosis levels of chronic liver diseases, including NASH. ${ }^{31}$ High LSM values in the NAFLD patients with CAP $\geq 264 \mathrm{~dB} / \mathrm{m}$ might indicate higher levels of fibrosis in this population, relating to the possible presence of NASH. However, the other serum fibrosis markers did not show significant differences between groups. A recent report showed the possibility of overestimating fibrosis among individuals with high CAP values, especially among those with low fibrosis stages. ${ }^{32}$ In addition, high body mass index may falsely increase LSM values in obese individuals. ${ }^{33}$ Evaluation of liver fibrosis with TE in this population (almost healthy individuals with at best only a few metabolic complications), should thus be considered carefully.

Nine patients $(8.8 \%$ of the 102 patients with NAFLD) showed values of $\geq 264 \mathrm{~dB} / \mathrm{m}$ and $\geq 7.0 \mathrm{kPa}$ from $\mathrm{TE}$, leading to suspicion of NASH. Abnormal HA was the most frequent finding ( 7 of 9 patients) among the fibrosis markers, followed by M2BPGi (4 of 9), NAFLD fibrosis score (3 of 9), and Fib-4 (1 of 9) (Table 3). Because serum HA can be elevated after exercise or by the presence of joint disease, these values should be interpreted with caution. Mizuno et al. ${ }^{34}$ reported that type IV collagen $7 \mathrm{~S}$ was more useful than HA in detecting early fibrosis of NASH. The diagnostic significance of M2BPGi in diagnosing NASH seemed to be confirmed by our cut-off index for M2BPGi to reveal early fibrosis of around 0.7. ${ }^{14,35}$ Referring to this value, 4 patients (3.9\% of the 102 NAFLD patients) fulfilled the criteria for LSM $\geq 7 \mathrm{kPa}$ and M2BPGi $>0.70$. Liver biopsy could be considered particularly in these 4 patients (3.9\%).

This study has some limitations. First, this was a crosssectional study that analyzed patients who visited our hospital during a certain period, so selection bias may have been present. Second, this study only examined the incidence of patients with a high index of suspicion for NASH among an almost-normal population without confirming the diagnosis by liver biopsy, and further studies are needed to validate these findings.

In conclusion, a significant correlation was seen between CAP values by TE and the presence of IR, showing that fat infiltration in the liver may be a trigger for inducing IR. About $9 \%$ of the Japanese population with NAFLD with a few metabolic complications could be suitable targets for liver biopsy to confirm NASH, including 3.9\% with M2BPGi $>0.70$. In addition to $T E$, use of other serological tests including M2BPGi may strengthen the decision making process for biopsy. 


\section{Acknowledgments}

This study was supported by a Grant-in-Aid for Scientific Research (C) (16K09564 to HH) from the Japan Society for the Promotion of Science (JSPS).

\section{Conflict of interest}

The authors have no conflict of interests related to this publication.

\section{Author contributions}

Collection and summarization of the data $(\mathrm{HH})$, critical discussions regarding the data ( $\mathrm{KW}$ and $\mathrm{KH})$, writing of the paper (SO).

\section{References}

[1] Vernon G, Baranova A, Younossi ZM. Systematic review: the epidemiology and natural history of non-alcoholic fatty liver disease and non-alcoholic steatohepatitis in adults. Aliment Pharmacol Ther 2011;34:274-285. doi: $10.1111 / j .1365-2036.2011 .04724 . x$

[2] Estes C, Razavi H, Loomba R, Younossi Z, Sanyal AJ. Modeling the epidemic of nonalcoholic fatty liver disease demonstrates an exponential increase in burden of disease. Hepatology 2018;67:123-133. doi: 10.1002/hep.29466.

[3] Kabbany MN, Conjeevaram Selvakumar PK, Watt K, Lopez R, Akras Z, Zein N et al. Prevalence of nonalcoholic steatohepatitis-associated cirrhosis in the United States: An analysis of national health and nutrition examination survey data. Am J Gastroenterol 2017;112:581-587. doi: 10.1038/ajg. 2017.5.

[4] Tilg H, Moschen AR, Roden M. NAFLD and diabetes mellitus. Nat Rev Gastroenterol Hepatol 2017;14:32-42. doi: 10.1038/nrgastro.2016.147.

[5] Karlas T, Petroff D, Sasso M, Fan JG, Mi YQ, de Lédinghen $V$, et al. Individual patient data meta-analysis of controlled attenuation parameter (CAP) technology for assessing steatosis. J Hepatol 2017;66:1022-1030. doi: 10. 1016/j.jhep.2016.12.022.

[6] Sasso M, Beaugrand $M$, de Ledinghen $V$, Douvin C, Marcellin P, Poupon $R$, et al. Controlled attenuation parameter (CAP): a novel VCTE ${ }^{\mathrm{TM}}$ guided ultrasonic attenuation measurement for the evaluation of hepatic steatosis: preliminary study and validation in a cohort of patients with chronic liver disease from various causes. Ultrasound Med Biol 2010;36:1825-1835. doi: 10. 1016/j.ultrasmedbio.2010.07.005.

[7] de Lédinghen V, Vergniol J, Capdepont M, Chermak F, Hiriart JB, Cassinotto C, et al. Controlled attenuation parameter (CAP) for the diagnosis of steatosis: a prospective study of 5323 examinations. J Hepatol 2014;60:1026-1031. doi: $10.1016 / j$.jhep.2013.12.018.

[8] Papagianni M, Sofogianni A, Tziomalos K. Non-invasive methods for the diagnosis of nonalcoholic fatty liver disease. World J Hepatol 2015;7:638-648. doi: 10.4254 /wjh.v7.i4.638

[9] Stern C, Castera L. Non-invasive diagnosis of hepatic steatosis. Hepatol Int 2017;11:70-78. doi: 10.1007/s12072-016-9772-z.

[10] Koda M, Matunaga Y, Kawakami M, Kishimoto Y, Suou T, Murawaki Y. FibroIndex, a practical index for predicting significant fibrosis in patients with chronic hepatitis C. Hepatology 2007;45:297-306. doi: 10.1002/hep.21520.

[11] Shah AG, Lydecker A, Murray K, Tetri BN, Contos MJ, Sanyal AJ. Comparison of noninvasive markers of fibrosis in patients with nonalcoholic fatty liver disease. Clin Gastroenterol Hepatol 2009;7:1104-1112. doi: 10.1016/j. cgh.2009.05.033.

[12] Angulo P, Hui JM, Marchesini G, Bugianesi E, George J, Farrell GC, et al. The NAFLD fibrosis score: a noninvasive system that identifies liver fibrosis in patients with NAFLD. Hepatology 2007;45:846-854. doi: 10.1002/hep. 21496

[13] Fitzpatrick E, Dhawan A. Noninvasive biomarkers in non-alcoholic fatty liver disease: current status and a glimpse of the future. World J Gastroenterol 2014;20:10851-10863. doi: 10.3748/wjg.v20.i31.10851.

[14] Abe M, Miyake T, Kuno A, Imai Y, Sawai Y, Hino K, et al. Association between Wisteria floribunda agglutinin-positive Mac-2 binding protein and the fibrosis stage of non-alcoholic fatty liver disease. J Gastroenterol 2015;50:776-784. doi: $10.1007 / \mathrm{s} 00535-014-1007-2$.

[15] Kuno A, Ikehara Y, Tanaka Y, Ito K, Matsuda A, Sekiya S, et al. A serum "sweet-doughnut" protein facilitates fibrosis evaluation and therapy assessment in patients with viral hepatitis. Sci Rep 2013;3:1065. doi: 10. 1038/srep01065.
[16] Lai LL, Chan WK, Sthaneshwar P, Nik Mustapha NR, Goh KL, Mahadeva S. Serum Wisteria floribunda agglutinin-positive Mac-2 binding protein in nonalcoholic fatty liver disease. PLoS One 2017;12:e0174982. doi: 10. 1371/journal.pone.0174982.

[17] Yamasaki K, Tateyama M, Abiru S, Komori A, Nagaoka S, Saeki A, et al. Elevated serum levels of Wisteria floribunda agglutinin-positive human Mac-2 binding protein predict the development of hepatocellular carcinoma in hepatitis $C$ patients. Hepatology 2014;60:1563-1570. doi: 10.1002/hep.27305.

[18] Yoneda M, Yoneda M, Mawatari H, Fujita K, Endo $H$, Iida $H$, et al. Noninvasive assessment of liver fibrosis by measurement of stiffness in patients with nonalcoholic fatty liver disease (NAFLD). Dig Liver Dis 2008;40:371-378. doi: 10.1016/j.dld.2007.10.019

[19] Yoshioka K, Hashimoto S, Kawabe N. Measurement of liver stiffness as a noninvasive method for diagnosis of non-alcoholic fatty liver disease. Hepatol Res 2015;45:142-151. doi: 10.1111/hepr.12388.

[20] Perry RJ, Samuel VT, Petersen KF, Shulman GI. The role of hepatic lipids in hepatic insulin resistance and type 2 diabetes. Nature 2014;510:84-91. doi: 10.1038/nature13478.

[21] Chon YE, Kim KJ, Jung KS, Kim SU, Park JY, Kim do Y, et al. The relationship between type 2 diabetes mellitus and non-alcoholic fatty liver disease measured by controlled attenuation parameter. Yonsei Med J 2016;57:885-892. doi: $10.3349 / y m j .2016 .57 .4 .885$

[22] de Lédinghen V, Wong GL, Vergniol J, Chan HL, Hiriart JB, Chan AW, et al. Controlled attenuation parameter for the diagnosis of steatosis in non-alcoholic fatty liver disease. J Gastroenterol Hepatol 2016;31:848-855. doi: 10. 1111 /jgh.13219.

[23] Masaki K, Takaki S, Hyogo H, Kobayashi T, Fukuhara T, Naeshiro N, et al. Utility of controlled attenuation parameter measurement for assessing liver steatosis in Japanese patients with chronic liver diseases. Hepatol Res 2013; 43:1182-1189. doi: 10.1111/hepr.12094.

[24] Liu K, Wong VW, Lau K, Liu SD, Tse YK, Yip TC, et al. Prognostic value of controlled attenuation parameter by transient elastography. Am J Gastroenterol 2017;112:1812-1823. doi: 10.1038/ajg.2017.389.

[25] Ferraioli G, Calcaterra V, Lissandrin R, Guazzotti M, Maiocchi L, Tinelli C, et al. Noninvasive assessment of liver steatosis in children: the clinical value of controlled attenuation parameter. BMC Gastroenterol 2017;17:61. doi: 10 . 1186/s12876-017-0617-6.

[26] Lee HW, Park SY, Kim SU, Jang JY, Park H, Kim JK, et al. Discrimination of nonalcoholic steatohepatitis using transient elastography in patients with nonalcoholic fatty liver disease. PLoS One 2016;11:e0157358. doi: 10. 1371/journal.pone. 0157358 .

[27] Yamazaki H, Tsuboya T, Tsuji K, Dohke M, Maguchi H. Independent association between improvement of nonalcoholic fatty liver disease and reduced incidence of type 2 diabetes. diabetes Care 2015;38:1673-1679. doi: 10 . 2337/dc15-0140.

[28] Okanoue T, Umemura A, Yasui K, Itoh Y. Nonalcoholic fatty liver disease and nonalcoholic steatohepatitis in Japan. J Gastroenterol Hepatol 2011;26 Suppl 1:153-162. doi: 10.1111/j.1440-1746.2010.06547.x.

[29] Aller de la Fuente R, Mora Cuadrado N, Tafur C, López Gómez JJ, Gómez de la Cuesta S, García Sánchez MC, et al. Histopathological differences in patients with biopsy-proven non-alcoholic fatty liver disease with and without type 2 diabetes. Endocrinol Diabetes Nutr 2018;65:354-360. doi: 10.1016/j. endinu.2017.12.011.

[30] Loomba R, Abraham M, Unalp A, Wilson L, Lavine J, Doo E, et al. Association between diabetes, family history of diabetes, and risk of nonalcoholic steatohepatitis and fibrosis. Hepatology 2012;56:943-951. doi: 10.1002/hep. 25772.

[31] Boursier J, Vergniol J, Guillet A, Hiriart JB, Lannes A, Le Bail B, et al. Diagnostic accuracy and prognostic significance of blood fibrosis tests and liver stiffness measurement by FibroScan in non-alcoholic fatty liver disease. J Hepatol 2016;65:570-578. doi: 10.1016/j.jhep.2016.04.023.

[32] Petta S, Wong VW, Cammà C, Hiriart JB, Wong GL, Marra F, et al. Improved noninvasive prediction of liver fibrosis by liver stiffness measurement in patients with nonalcoholic fatty liver disease accounting for controlled attenuation parameter values. Hepatology 2017;65:1145-1155. doi: 10 . 1002/hep.28843.

[33] Mikolasevic I, Orlic L, Franjic N, Hauser G, Stimac D, Milic S. Transient elastography (FibroScan $\left({ }^{\circledR}\right)$ ) with controlled attenuation parameter in the assessment of liver steatosis and fibrosis in patients with nonalcoholic fatty liver disease - Where do we stand? World J Gastroenterol 2016;22:72367251. doi: 10.3748/wjg.v22.i32.7236.

[34] Mizuno M, Shima T, Oya H, Mitsumoto Y, Mizuno C, Isoda S, et al. Classification of patients with non-alcoholic fatty liver disease using rapid immunoassay of serum type IV collagen compared with liver histology and other fibrosis markers. Hepatol Res 2017;47:216-225. doi: 10.1111/hepr.12710.

[35] Nishikawa H, Enomoto H, Iwata Y, Kishino K, Shimono Y, Hasegawa K, et al. Clinical significance of serum Wisteria floribunda agglutinin positive Mac-2binding protein level in non-alcoholic steatohepatitis. Hepatol Res 2016;46: 1194-1202. doi: 10.1111/hepr.12662. 